\title{
A COMPENDIUM OF STRATEGIES TO PREVENT HEALTHCARE-ASSOCIATED INFECTIONS IN ACUTE CARE HOSPITALS
}

Introduction: Improving Patient Safety Through Infection Control: A New Healthcare Imperative • Deborah S. Yokoe, MD, MPH; David Classen, MD, MS

\section{SHEA/IDSA PRACTICE RECOMMENDATIONS}

Executive Summary: A Compendium of Strategies to Prevent Healthcare-Associated Infections in Acute Care Hospitals - Deborah S. Yokoe, MD, MPH; Leonard A. Mermel, DO, ScM; Deverick J. Anderson, MD, MPH; Kathleen M. Arias, MS, CIC; Helen Burstin, MD; David P. Calfee, MD, MS; Susan E. Coffin, MD; Erik R. Dubberke, MD; Victoria Fraser, MD; Dale N. Gerding, MD; Frances A. Griffin, RRT, MPA; Peter Gross, MD; Keith S. Kaye, MD; Michael Klompas, MD; Evelyn Lo, MD; Jonas Marschall, MD; Lindsay Nicolle, MD; David A. Pegues, MD; Trish M. Perl, MD; Kelly Podgorny, RN, MS, CPHQ; Sanjay Saint, MD; Cassandra D. Salgado, MD, MS; Robert A. Weinstein, MD; Robert Wise, MD; David Classen, MD, MS

Strategies to Prevent Central Line-Associated Bloodstream Infections in Acute Care Hospitals • Jonas Marschall, MD; Leonard A. Mermel, DO, ScM; David Classen, MD, MS; Kathleen M. Arias, MS, CIC; Kelly Podgorny, RN, MS, CPHQ; Deverick J. Anderson, MD, MPH; Helen Burstin, MD; David P. Calfee, MD, MS; Susan E. Coffin, MD, MPH; Erik R. Dubberke, MD; Victoria Fraser, MD; Dale N. Gerding, MD; Frances A. Griffin, RRT, MPA; Peter Gross, MD; Keith S. Kaye, MD; Michael Klompas, MD; Evelyn Lo, MD; Lindsay Nicolle, MD; David A. Pegues, MD; Trish M. Perl, MD; Sanjay Saint, MD; Cassandra D. Salgado, MD, MS; Robert A. Weinstein, MD; Robert Wise, MD; Deborah S. Yokoe, MD, MPH

\section{Strategies to Prevent Ventilator-Associated Pneumonia in Acute Care Hospitals •}

Susan E. Coffin, MD, MPH; Michael Klompas, MD; David Classen, MD, MS; Kathleen M. Arias, MS, CIC; Kelly Podgorny, RN, MS, CPHQ; Deverick J. Anderson, MD, MPH; Helen Burstin, MD; David P. Calfee, MD, MS; Erik R. Dubberke, MD; Victoria Fraser, MD; Dale N. Gerding, MD; Frances A. Griffin, RRT, MPA; Peter Gross, MD; Keith S. Kaye, MD; Evelyn Lo, MD; Jonas Marschall, MD; Leonard A. Mermel, DO, ScM; Lindsay Nicolle, MD; David A. Pegues, MD; Trish M. Perl, MD; Sanjay Saint, MD; Cassandra D. Salgado, MD, MS; Robert A. Weinstein, MD; Robert Wise, MD; Deborah S. Yokoe, MD, MPH

\section{Strategies to Prevent Catheter-Associated Urinary Tract Infections in Acute Care Hospitals •}

Evelyn Lo, MD; Lindsay Nicolle, MD; David Classen, MD, MS; Kathleen M. Arias, MS, CIC;

Kelly Podgorny, RN, MS, CPHQ; Deverick J. Anderson, MD, MPH; Helen Burstin, MD; David P. Calfee, MD, MS; Susan E. Coffin, MD, MPH; Erik R. Dubberke, MD; Victoria Fraser, MD; Dale N. Gerding, MD;

Frances A. Griffin, RRT, MPA; Peter Gross, MD; Keith S. Kaye, MD; Michael Klompas, MD; Jonas Marschall, MD; Leonard A. Mermel, DO, ScM; David A. Pegues, MD; Trish M. Perl, MD; Sanjay Saint, MD; Cassandra D. Salgado, MD, MS; Robert A. Weinstein, MD; Robert Wise, MD; Deborah S. Yokoe, MD, MPH 


\section{INFECTION CONTROL \& HOSPITAL EPIDEMIOLOGY}

CONTENTS CONTINUED FROM COVER

S51 Strategies to Prevent Surgical Site Infections in Acute Care Hospitals • Deverick J. Anderson, MD, MPH; Keith S. Kaye, MD; David Classen, MD, MS; Kathleen M. Arias, MS, CIC; Kelly Podgorny, RN, MS, CPHQ; Helen Burstin, MD; David P. Calfee, MD, MS; Susan E. Coffin, MD, MPH; Erik R. Dubberke, MD; Victoria Fraser, MD; Dale N. Gerding, MD; Frances A. Griffin, RRT, MPA; Peter Gross, MD; Michael Klompas, MD; Evelyn Lo, MD; Jonas Marschall, MD; Leonard A. Mermel, DO, ScM; Lindsay Nicolle, MD; David A. Pegues, MD; Trish M. Perl, MD; Sanjay Saint, MD; Cassandra D. Salgado, MD, MS; Robert A. Weinstein, MD; Robert Wise, MD; Deborah S. Yokoe, MD, MPH

S62 Strategies to Prevent Transmission of Methicillin-Resistant Staphylococcus aureus in Acute Care Hospitals - David P. Calfee, MD, MS; Cassandra D. Salgado, MD, MS; David Classen, MD, MS; Kathleen M. Arias, MS, CIC; Kelly Podgorny, RN, MS, CPHQ; Deverick J. Anderson, MD, MPH; Helen Burstin, MD; Susan E. Coffin, MD, MPH; Erik R. Dubberke, MD; Victoria Fraser, MD; Dale N. Gerding, MD; Frances A. Griffin, RRT, MPA; Peter Gross, MD; Keith S. Kaye, MD; Michael Klompas, MD; Evelyn Lo, MD; Jonas Marschall, MD; Leonard A. Mermel, DO, ScM; Lindsay Nicolle, MD; David A. Pegues, MD; Trish M. Perl, MD; Sanjay Saint, MD; Robert A. Weinstein, MD; Robert Wise, MD; Deborah S. Yokoe, MD, MPH

S81 Strategies to Prevent Clostridium difficile Infections in Acute Care Hospitals - Erik R. Dubberke, MD; Dale N. Gerding, MD; David Classen, MD, MS; Kathleen M. Arias, MS, CIC; Kelly Podgorny, RN, MS, CPHQ; Deverick J. Anderson, MD, MPH; Helen Burstin, MD; David P. Calfee, MD, MS; Susan E. Coffin, MD, MPH; Victoria Fraser, MD; Frances A. Griffin, RRT, MPA; Peter Gross, MD; Keith S. Kaye, MD; Michael Klompas, MD; Evelyn Lo, MD; Jonas Marschall, MD; Leonard A. Mermel, DO, ScM; Lindsay Nicolle, MD; David A. Pegues, MD; Trish M. Perl, MD; Sanjay Saint, MD; Cassandra D. Salgado, MD, MS; Robert A. Weinstein, MD; Robert Wise, MD; Deborah S. Yokoe, MD, MPH 
An Official Publication of the Society for Healthcare Epidemiology of America

\section{EDITOR}

Suzanne F. Bradley, MD • Ann Arbor, MI

\section{DEPUTY EDITOR}

Carol A. Kauffman, MD $\cdot$ Ann Arbor, MI

\section{SENIOR ASSOCIATE EDITORS}

C. Glen Mayhall, MD • Galveston, TX

Gina Pugliese, RN, MS • Chicago, IL

William Schaffner, MD $・$ Nashville, TN

\section{ASSOCIATE EDITORS}

Ebbing Lautenbach, MD, MPH • Philadelphia, PA

Preeti N. Malani, MD, MSI - Ann Arbor, MI

David Weber, MD, MPH $\bullet$ Chapel Hill, NC

\section{STATISTICS CONSULTANT}

Rodney L. Dunn, MS • Ann Arbor, MI

SECTION EDITOR FOR GUIDELINES, POSITION PAPERS, AND INVITED REVIEWS

Carol Chenoweth, MD • Ann Arbor, MI

\section{MANAGING EDITOR}

Gordon Rudy, PhD, ELS • Chicago, IL

\section{PAST EDITORS}

\section{Infection Control}

Richard P. Wenzel, MD, 1980-1990 (vols. 1-11)

Infection Control and Hospital Epidemiology

Richard P. Wenzel, MD, 1991-1992 (vols. 12 and 13)

Michael D. Decker, MD, 1993-2001 (vols. 14-22)

Barry M. Farr, MD, 2002-2004 (vols. 23-25)

William R. Jarvis, MD, 2005-2006 (vols. 26 and 27)

\section{EDITORIAL ADVISORY BOARD}

Miriam Alter, $\mathrm{PhD}, \mathrm{MD} \cdot$ Atlanta, $\mathrm{GA}$ Lennox Archibald, MD, FRCP • Alachua, FL Hilary Babcock, MD - St. Louis, MO Elise M. Beltrami, MD, MPH - Decatur, GA David Birnbaum, $\mathrm{PhD}, \mathrm{MPH} \cdot$ Sidney, BC Christian Brun-Buisson, MD - Creteil, France John P. Burke, MD • Salt Lake City, UT David P. Calfee, MD, MS • New York, NY Yehuda Carmeli, MD, MPH $\cdot$ Tel Aviv, Israel Sara E. Cosgrove, MD, MS $\bullet$ Baltimore, MD Donald E. Craven, $\mathrm{MD} \cdot$ Burlington, MA Burke A. Cunha, MD $\cdot$ Mineola, NY Erika D'Agata, MD, MPH $\bullet$ Boston, MA Patch Dellinger, $M D \cdot$ Seattle, WA

Daniel Diekema, MD $\cdot$ Iowa City, IA Charles E. Edmiston, Jr., $\mathrm{PhD} \cdot$ Milwaukee, WI Theodore C. Eickhoff, MD $\bullet$ Denver, CO Martin S. Favero, $\mathrm{PhD} \cdot$ Irvine, $\mathrm{CA}$ Richard A. Garibaldi, MD - Farmington, CT Petra Gastmeier, MD $\cdot$ Hanover, Germany Dale N. Gerding, MD $\cdot$ Hines, IL

Donald A. Goldmann, MD • Boston, MA Anthony D. Harris, MD, MPH - Baltimore, MD David K. Henderson, MD $\bullet$ Bethesda, MD Loreen A. Herwaldt, MD - Iowa City, IA Peter N. R. Heseltine, MD • San Juan, CA Karen Hoffmann, RN, CIC, MS • Chapel Hill, NC Janine Jagger, $\mathrm{MPH}, \mathrm{PhD} \cdot$ Charlottesville, VA John A. Jernigan, MD, MS $\bullet$ Atlanta, GA James T. Lee, $\mathrm{MD}, \mathrm{PhD} \cdot$ St. Paul, $\mathrm{MN}$ L. Clifford McDonald, MD • Atlanta, GA Allison McGeer, MD • Toronto, ON
Leonard A. Mermel, DO, ScM - Providence, RI Robert R. Muder, MD - Pittsburgh, PA Carlene A. Muto, MD, MS - Pittsburgh, PA Joseph M. Mylotte, MD, CIC $\cdot$ Buffalo, NY David L. Paterson, MBBS, FRACP - Pittsburgh, PA Jan Evans Patterson, MD - San Antonio, TX Sindy M. Paul, MD - Yardley, PA David A. Pegues, MD • Los Angeles, CA Didier Pittet, MD, MS • Geneva, Switzerland Michael A. Pfaller, MD • Iowa City, IA Gina Pugliese, RN, MS • Chicago, IL Isaam Raad, MD $\cdot$ Houston, TX Jordi Rello, $\mathrm{MD}, \mathrm{PhD} \cdot$ Tarragona, Spain Manfred L. Rotter, MD, DipBact • Vienna, Austria Henning Rüden, MD • Berlin, Germany William A. Rutala, PhD, MPH $\bullet$ Chapel Hill, NC Lisa Saiman, MD, MPH • New York, NY William E. Scheckler, MD • Madison, WI Lynne M. Sehulster, $\mathrm{PhD} \cdot$ Atlanta, GA John A. Sellick, DO • Amherst, NY Kent Sepkowitz, MD $•$ New York, NY Andrew E. Simor, MD - Toronto, ON Philip W. Smith, MD - Omaha, NE Denis W. Spelman, MD - Prahan, Victoria, Australia Jeffrey R. Starke, MD $\cdot$ Houston, TX Janet E. Stout, $\mathrm{PhD}$ - Pittsburgh, $\mathrm{PA}$ Clyde Thornsberry, $\mathrm{PhD} \cdot$ Franklin, TN William Trick, MD • Chicago, IL Antoni Trilla, $\mathrm{MD}, \mathrm{PhD} \cdot$ Barcelona, Spain Robert A. Weinstein, MD $\cdot$ Chicago, IL Andreas Widmer, MD, MS • Basel, Switzerland Marcus Zervos, MD $\cdot$ Royal Oak, MI
Infection Control and Hospital Epidemiology (ISSN 0899-823X) is published monthly by the University of Chicago Press, 1427 E. 60th St., Chicago, IL 60637-2954 (http:// www.journals.uchicago.edu/ICHE/). The editorial office is in Chicago, Illinois.

\section{Editorial Office}

Communications should be addressed to the Editor, Infection Control and Hospital Epidemiology, 1427 E. 60th St., Chicago, IL 60637-2954; (e-mail: iche@press.uchicago.edu; telephone: 773-702-2448, fax: 773-753-4247). Contributors should consult the Information for Authors, which is available at the journal's Web site.

\section{Advertising}

Please direct advertising inquiries to Journals Advertising, University of Chicago Press, 1427 E. 60th St., Chicago, IL 60637 (e-mail: thill@press.uchicago.edu; telephone: 773-7028187; fax: 773-702-0172). Publication of an advertisement in Infection Control and Hospital Epidemiology does not imply endorsement of its claims by the Society for Healthcare Epidemiology of America, by the Editor, or by the University of Chicago. Correspondence regarding advertising should be addressed to the advertising office in Chicago.

\section{Permissions}

Articles may be copied or otherwise reused without permission only to the extent permitted by Sections 107 and 108 of the US Copyright Law. Permission to copy articles for personal, internal, classroom, or library use may be obtained from the Copyright Clearance Center (http://www.copyright.com). For all other uses, such as copying for general distribution, for advertising or promotional purposes, for creating new collective works, or for resale, please contact the Permissions Coordinator, Journals Division, University of Chicago Press, 1427 E. 60th St., Chicago, IL 60637 (e-mail: journalpermissions@press.uchicago.edu; fax: 773-834-3489). Articles in the public domain may be used without permission, but it is customary to contact the author.

\section{Subscriptions}

Subscription rates for 2008 are $\$ 164$ for individuals and $\$ 386$ for institutions. Additional rates for non-US subscribers and reduced rates for fellows, residents, and students are available at http://www.journals.uchicago.edu/ICHE. Subscription agent for Japan: Kinokuniya Company, Ltd. Individuals have the option to order directly from the University of Chicago Press. Single copy rates: individuals $\$ 17$; institutions $\$ 38$.

Please direct subscription inquries, requests for back issues, and address changes to Journals Division, University of Chicago Press, P.O. Box 37005, Chicago, IL 60637 (e-mail: subscriptions@press.uchicago.edu; telephone: 773-753-3347 or toll-free in the United States and Canada 877-705-1878; fax: 773-753-0811 or toll-free 877-705-1879).

Published by the University of Chicago Press, Chicago, Illinois, (C) 2008 by the Society for Healthcare Epidemiology of America. All rights reserved. This publication is printed on acid-free paper. 


\section{Sponsorship Acknowledgment}

This compendium of practice recommendations was sponsored and authored by the Society for Healthcare Epidemiology of America (SHEA) and the Infectious Diseases Society of America (IDSA). Partners in this work were the Association for Professionals in Infection Control and Epidemiology (APIC), the Joint Commission, and the American Hospital Association (AHA). 